\section{Fatores associados à indução do parto em gestantes com óbito fetal após a $20^{\mathrm{a}}$ semana}

\section{Factors associated with induced delivery in pregnant women with a dead fetus after the 20th week of gestation}

Ânderson Gonçalves Sampaio 1

Alex Sandro Rolland Souza 2

Carlos Noronha Neto 3

\begin{abstract}
Objectives: to determine the factors associated with induced delivery in pregnant women carrying a dead fetus.

Methods: a cross-sectional study with 258 pregnant women past the 20th week of gestation diagnosed by ultrasound as carrying a dead fetus, between January 2005 and December 2008, at the Hospital Barão de Lucena maternity hospital, in Recife, Brazil. Socio-demographic characteristics, causes and types of fetal death, prior obstetric data and characteristics of birth were the variables studied. The chi-square test, Fisher's exact test and Student's t test were used. The rate of prevalence was calculated and multiple logistic regression was carried out. The level of significance was $5 \%$.

Results: birth was induced in 83 (32.2\%) of the pregnant women. Following multivariate analysis, it was found that a gestational age of over 40 weeks $(O R=126.5 ; C 195 \%=3.83-4,201.5)$ and late fetal death $(O R=6.86 ; C 195 \%=2.55-18.47)$ were the risk factors that continued to be associated with induced delivery. Caesarian section $(O R=0.02 ; C 195 \%=0.004-0.09)$, funiculopathy $(O R=0.12 ; C 195 \%=0.02-0.68)$, having had one or more previous pregnancies $(O R=0.34 ; C 195 \%=$ 0.14-0.81) and one or more previous caesarian sections $(O R=0.16 ;$ IC95\%=0.04-0.71) were negatively associated with induced delivery.

Conclusions: a gestational age of over 40 weeks and late fetal death are the risk factors that could be associated with induced delivery in pregnant women carrying a dead fetus.

Key words Labor induced, Fetal death, Stillbirth, Cesarean section
\end{abstract}

${ }^{1}$ Setor de Tocoginecologia. Hospital Barão de Lucena. Recife, PE, Brasil.

2,3 Centro de Atenção à Mulher. Instituto de Medicina Integral Prof. Fernando Figueira. Rua dos Coelhos, 300. Boa Vista. Recife, PE, Brasil. CEP: 50.070-550. E-mail: alexrolland@uol.com.br

\section{Resumo}

Objetivos: determinar os fatores associados à indução do trabalho de parto em gestantes com óbito fetal.

Métodos: estudo seccional, com 258 gestantes após a $20^{a}$ semana e diagnóstico ultrassonográfico de óbito fetal, no periodo de janeiro/2005 a dezembro/2008, na maternidade do Hospital Barão de Lucena, Recife, Brasil. Características sociodemográficas, causas e tipos de óbito fetal, antecedentes obstétricos e características do parto foram variáveis estudadas. Foram utilizados testes qui-quadrado, exato de Fisher e " $t$ " de Student. Razão de prevalência e análise de regressão logística múltipla também foram realizados. O nivel de significância foi de 5\%.

Resultados: a indução do parto ocorreu em 83 (32,2\%) gestantes. Após análise multivariada, observou-se que a idade gestacional acima da $40^{a}$ semana de gravidez $(O R=126,5 ;$ IC95\% $=3,83$ $4.201,5)$ e óbito fetal tardio $(O R=6,86 ; 1 C 95 \%=$ 2,55-18,47) foram fatores de risco que permaneceram associados à indução do parto. Cesariana $(O R=0,02 ; I C 95 \%=0,004-0,09)$, funiculopatias $(O R=0,12 ;$ IC95\%=0,02-0,68), presença de uma ou menos gestações anteriores $(O R=0,34 ;$ IC95\%=0,14-0,81) e uma ou mais cesáreas anteriores $(O R=0,16 ; 1 C 95 \%=0,04$ $0,71)$ foram negativamente associados à indução.

Conclusões: idade gestacional acima da $40^{a}$ semana e óbito fetal tardio são fatores de risco que podem estar associados à indução do parto em gestantes com óbito fetal.

Palavras-chave Trabalho de parto induzido, Morte fetal, Natimorto, Cesárea 


\section{Introdução}

Nos últimos anos, devido aos avanços nos recursos propedêuticos, a prática da indução do trabalho de parto tem tido maior utilização entre obstetras, principalmente em hospitais de referência para gestação de alto risco. ${ }^{1-5}$ A preocupação com o excesso de cesarianas, mesmo em pacientes com fetos mortos, tem trazido crescente interesse pelo tema. 1

A indução do parto consiste no estímulo artificial às contrações uterinas antes do início espontâneo, em gestantes acima da $22^{\mathrm{a}}$ semana. O desencadeamento do processo de parturição deverá ocorrer de forma coordenada e efetiva, culminando com o parto vaginal. 6

Entre os métodos naturais existe a estimulação mamária, além dos artificiais, como mecânicos 3,4 ou medicamentosos.5,7-10 Os dilatadores cervicais osmóticos (laminária), não disponíveis para comercialização no Brasil, e os cateteres extra-ovulares como a sonda de Foley, utilizada em pacientes com cesariana anterior, fazem parte dos métodos mecânicos de indução. 4 Também são empregados o descolamento de membranas do pólo inferior fetal e a ruptura artificial de membranas ovulares. A administração de solução hipertônica foi abandonada em razão da alta morbidade materna detectada em estudos de bases experimentais. No entanto, admitese que tais procedimentos sejam limitados por exigirem dilatação cervical prévia e não terem boa aceitação pelas usuárias. 3,4

Diversos medicamentos fazem parte do arsenal obstétrico, dentre os quais a ocitocina, que possui baixa efetividade quando o colo uterino se mantém com escore de Bishop desfavorável, e as prostaglandinas, como o misoprostol e o mifepristone. $5,8-10$

Em gestantes com feto morto, a tendência atual é a conduta ativa, devido ao quadro de ansiedade da mãe e pela possibilidade da ocorrência de coagulação intravascular disseminada quando há retenção do feto morto. ${ }^{7}$ Estudos sugerem alta efetividade e eficácia do misoprostol para indução do parto em gestantes com feto morto. ${ }^{11-13}$

Quanto aos fatores associados à indução, em gestantes com fetos vivos, foram encontrados apenas dois estudos, realizados na America Latina sendo um deles no Brasil. ${ }^{14,15}$ Sugere-se que os fatores preditivos mais importantes para parto vaginal após indução com misoprostol são o escores de Bishop maior ou igual a quatro e o antecedente de um ou mais partos. ${ }^{14} \mathrm{Em}$ contrapartida, não há descrições disponíveis em pacientes com fetos mortos, provavelmente porque o trabalho de parto é induzido na maioria das gestações $(73,0 \%) .7$
Atualmente, os fatores preditivos mais importantes para parto vaginal, após indução com misoprostol, são paridade maior ou igual a um e escore de Bishop maior ou igual a quatro. Estes fatores devem ser levados em consideração na escolha de diferentes esquemas e doses de misoprostol, tanto para preparo cervical como para indução do parto. 14

Com o objetivo de identificar pacientes com maior chance de êxito no processo de indução do trabalho de parto em gestantes com feto morto foi realizado o presente trabalho.

\section{Métodos}

Realizou-se estudo seccional em gestantes, com fetos mortos, internadas na maternidade de alto risco do Hospital Barão de Lucena (HBL) na cidade de Recife, Pernambuco, no período de janeiro de 2005 a dezembro de 2008.

Foram incluídas 258 pacientes com idade gestacional maior ou igual a $20^{\mathrm{a}}$ semana, peso do concepto ao nascimento maior ou igual a $500 \mathrm{~g}$ e confirmação ultrassonográfica de óbito fetal, segundo critérios da World Health Organization (WHO) 16 e o National Center for Health Statistic. 17

A ultrassonografia foi realizada a partir de queixa da paciente de ausência de movimentação fetal e/ou suspeita clínica de batimento cardíaco fetal não evidenciado pelo aparelho acústico sonar.

As variáveis analisadas foram método de indução de parto (misoprostol, ocitocina, ruptura artificial das membranas, descolamento das membranas e associação entre os métodos), via de parto da gestação atual (cesárea, vaginal e instrumental), idade materna (anos completos), idade gestacional (semanas completas), escolaridade (anos estudados), estado civil (com e sem companheiro), antecedentes obstétricos (gestações, abortos, partos vaginais e cesáreas anteriores), assistência pré-natal, tipo de gestação (única e gemelar), peso fetal (gramas), indicação da cesariana e causas e tipo do óbito.

A indução do parto foi definida como o uso de qualquer método capaz de estimular artificialmente contrações uterinas coordenadas e efetivas, antes de seu início espontâneo, levando ao desencadeamento do trabalho de parto. ${ }^{6}$ Grande parte da indução mecânica foi realizada por médicos assistentes.

A idade gestacional do parto, em semanas completas, foi calculada tendo como base a data da última menstruação, desde que conhecida, confiável e confirmada pela primeira ultrassonografia. Caso a gestante não soubesse informar a data da última menstruação, considerou-se a idade gestacional da 
primeira ultrassonografia. O peso fetal foi classificado em baixo peso, quando abaixo de $2500 \mathrm{~g}$ e macrossomia quando maior que $4000 \mathrm{~g}$.

O óbito fetal foi classificado de acordo com a idade gestacional em precoce, quando ocorreu entre a $20^{\mathrm{a}}$ e $28^{\mathrm{a}}$ semanas, e tardio, a partir da $28^{\mathrm{a}}$ semana completa. Classificaram-se ainda os óbitos intraútero de acordo com a época do acontecimento, em anteparto, quando o óbito do concepto ocorreu antes de iniciado a indução e/ou trabalho de parto, e intraparto, quando o óbito ocorreu após início da indução do parto (em feto vivo) e/ou trabalho de parto. 18

As causas dos óbitos fetais foram determinadas pela $10^{\mathrm{a}}$ classificação internacional de doenças (CID-10), ${ }^{19}$ sendo colhidas da declaração de óbito e ratificadas conforme descrições do prontuário médico, quando necessário.

As indicações de cesarianas foram identificadas nos relatórios cirúrgicos presentes nos prontuários médicos. As fontes de dados foram o sistema de informação sobre mortalidade (SIM) do HBL, fornecido pela secretaria de vigilância em saúde do Estado de Pernambuco, e avaliação dos prontuários do serviço com declarações de óbitos fetais e descrições dos procedimentos empregados.

Os dados foram coletados de forma retrospectiva pelo pesquisador, utilizando prontuários médicos de obstetrícia. Todas as pacientes, incluídas no estudo, tiveram seus diagnósticos confirmados no pós-parto e seguiram acompanhadas de acordo com a rotina do serviço, sem prejuízo nas condutas adotadas naquele momento.

As informações foram digitadas em banco de dados criado no programa estatístico Epi-Info 3.5.1. Realizaram-se análises comparativas das variáveis independentes com a necessidade ou não de indução do trabalho de parto. Em relação às variáveis quantitativas a comparação entre os grupos foi realizada pelo teste $t$ de Student. As variáveis categóricas foram comparadas em tabelas de contingência, utilizando-se testes qui-quadrado de associação e exato de Fisher, quando pertinente, a um nível de significância de 5\%. Calculou-se a razão de prevalência (RP) como medida de risco e seu intervalo de confiança a $95 \%$. Selecionaram-se variáveis independentes que apresentaram um nível de significância de $20 \%$ para realização da análise multivariada. Realizou-se análise de regressão logística múltipla stepwise, evidenciando-se, para o modelo final, variáveis que persistiram associadas com o desfecho.

O projeto de pesquisa foi aprovado pelo Comitê de Ética em Pesquisa do Hospital Agamenon Magalhães sob protocolo no 253.766, em 29/04/2009.

\section{Resultados}

Dos 258 óbitos fetais, houve necessidade de indução do trabalho de parto em 83 (32,2\%) gestações. O método de indução mais realizado foi o medicamentoso com o misoprostol $(\mathrm{n}=56 ; 67,5 \%)$, seguido de ocitocina $(n=9 ; 10,8 \%)$, misoprostol associado à ocitocina $(n=8 ; 9,6 \%)$, misoprostol associado à ruptura artificial das membranas $(n=7 ; 8,4 \%)$ e misoprostol associado ao descolamento das membranas $(n=3 ; 3,6 \%)$.

A idade materna variou entre 14 e 44 anos, com médias similares entre os grupos com e sem indução do trabalho de parto $(26,7 \pm 7,7$ anos $v s 25,8 \pm 7,2$ anos; respectivamente, $p=0,36$ ). De forma semelhante não houve diferença entre os grupos para o número de gestações $(2,4 \pm 2,7$ vs $2,1 \pm 2,4 ; p=0,29)$, partos $(1,8 \pm 2,2$ vs $1,5 \pm 2,1 ; p=0,28)$ ou abortos $(0,6 \pm 0,9$ vs $0,5 \pm 0,7 ; p=0,76)$ anteriores entre os grupos (Tabela 1).

No momento do parto, a idade gestacional variou

Tabela 1

Gestantes com óbito fetal do Hospital Barão de Lucena, Recife, no período de 2005 a 2008.

\begin{tabular}{|c|c|c|c|}
\hline \multirow{3}{*}{ Variáveis } & \multicolumn{2}{|c|}{ Indução do parto } & \multirow{3}{*}{$p^{*}$} \\
\hline & $\operatorname{Sim}(N=83)$ & Não (N=175) & \\
\hline & $\bar{x} \pm D P$ & $\bar{X} \pm D P$ & \\
\hline Idade materna (anos) & $26,7 \pm 7,7$ & $25,8 \pm 7,2$ & 0,36 \\
\hline Idade gestacional do parto (semanas) & $33,9 \pm 4,8$ & $29,8 \pm 5,6$ & $<0,01$ \\
\hline Número de gestações anteriores & $2,4 \pm 2,7$ & $2,1 \pm 2,4$ & 0,29 \\
\hline Número de partos anteriores & $1,8 \pm 2,2$ & $1,5 \pm 2,1$ & 0,28 \\
\hline Número de abortos anteriores & $0,6 \pm 0,9$ & $0,5 \pm 0,7$ & 0,76 \\
\hline Peso fetal (g) & $2356,0 \pm 1136,0$ & $1548,0 \pm 939,0$ & $<0,01$ \\
\hline
\end{tabular}

* Teste $\mathrm{t}$ de Student. 
entre a $20^{\mathrm{a}}$ e $42^{\mathrm{a}}$ semanas, sendo a média de $33,9 \pm$ 4,8 semanas no grupo de pacientes que realizaram indução do parto e $29,8 \pm 5,6$ semanas nas pacientes que não realizaram $(p<0,01)$. Houve diferença significativa entre os pesos dos fetos mortos nos grupos de gestantes com e sem indução para o trabalho de parto $(2356 \pm 1136 \mathrm{~g}$ vs. $1548 \pm 939 \mathrm{~g} ; p<0,01)$ (Tabela 1).

A via de parto cesariana foi realizada em 71 gestantes com óbito fetal, sendo indicada em sete $(9,9 \%)$ pacientes por falha de indução. As principais indicações do parto cirúrgico foram descolamento prematuro de placenta $(\mathrm{n}=35 ; 49,3 \%)$, síndromes hipertensivas $(n=10 ; 14,1 \%)$, duas ou mais cesarianas anteriores $(n=10 ; 14,1 \%)$, gestação múltipla $(\mathrm{n}=8 ; 11,3 \%)$ e apresentação pélvica e sofrimento fetal intraparto $(n=4 ; 5,6 \%)$

Não houve descrição da causa do óbito fetal em $83(31,4 \%)$ pacientes. Das 177 gestantes com feto morto, as síndromes hipertensivas ( $\mathrm{n}=77 ; 43,5 \%)$, descolamento prematuro da placenta $(n=44 ; 24,9 \%)$, infecções maternas $(\mathrm{n}=18 ; 10,2 \%)$, malformações congênitas $(n=16 ; 9,0 \%)$ e ruptura prematura das membranas $(n=14 ; 7,9 \%)$ foram as principais causas conhecidas.

$\mathrm{Na}$ análise bivariada, a ausência de companheiro $(p<0,01)$, se primigesta ou ter uma gestação anterior $(p=0,04)$, uma ou mais cesáreas anteriores $(p<0,01)$, ausência de parto vaginal anterior $(p=0,04)$, via de parto cesariana $(p<0,01)$ e baixo peso do feto no parto $(p<0,01)$ ocorreram em menor frequência nas gestantes que realizaram indução do parto. Enquanto, que óbito fetal tardio $(p<0,01)$, a idade gestacional do parto acima da $37^{\mathrm{a}}$ e $40^{\mathrm{a}}$ semana de gravidez $(p<0,01)$ e macrossomia fetal $(>4000 \mathrm{~g})$ $(p=0,01)$ ocorreram em maior frequência nas pacientes submetidas à indução do parto. A idade materna, a escolaridade, paridade, o número de abortos anteriores, a gestação múltipla, a assistência pré-natal e o óbito intraparto não apresentaram diferença significativa entre os grupos (Tabela 2).

Quanto às causas do óbito fetal, foram associ-

\section{Tabela 2}

Fatores associados à indução do parto em gestantes com óbito fetal do Hospital Barão de Lucena, Recife, no período de 2005 a 2008.

\begin{tabular}{|c|c|c|c|c|c|c|c|}
\hline \multirow{3}{*}{ Fatores associados } & \multicolumn{4}{|c|}{ Indução do parto } & \multirow{3}{*}{$\mathbf{R P}$} & \multirow{3}{*}{$\mathrm{IC} 95 \%$} & \multirow{3}{*}{$p^{*}$} \\
\hline & \multicolumn{2}{|c|}{$\operatorname{Sim}(N=83)$} & \multicolumn{2}{|c|}{ Não (N=175) } & & & \\
\hline & $\mathrm{n}$ & $\%$ & $\mathrm{n}$ & $\%$ & & & \\
\hline \multicolumn{8}{|l|}{ Idade materna (anos) } \\
\hline$\leq 19$ & 16 & 19,3 & 41 & 23,4 & 0,84 & $0,53-1,33$ & 0,45 \\
\hline$\geq 35$ & 17 & 20,5 & 25 & 14,3 & 1,32 & $0,87-2,01$ & 0,21 \\
\hline Escolaridade $\leq 3$ anos ** & 14 & 16,9 & 34 & 19,4 & 0,89 & $0,55-1,44$ & 0,62 \\
\hline Estado civil: sem companheiro & 28 & 33,7 & 94 & 53,7 & 0,57 & $0,37-0,83$ & $<0,01$ \\
\hline Gestações anteriores $\leq 1$ & 33 & 39,8 & 93 & 53,1 & 0,69 & $0,48-0,99$ & 0,04 \\
\hline Partos anteriores $<1$ & 27 & 32,5 & 65 & 37,1 & 0,87 & $0,59-1,27$ & 0,47 \\
\hline Abortos anteriores $<1$ & 46 & 55,4 & 94 & 53,5 & 1,05 & $0,73-1,49$ & 0,79 \\
\hline Cesárea anterior $\geq 1$ & 5 & 6,0 & 33 & 18,9 & 0,37 & $0,16-0,86$ & $<0,01$ \\
\hline Parto normal anterior $<1$ & 30 & 36,1 & 86 & 49,1 & 0,69 & $0,47-1,00$ & 0,04 \\
\hline Gestação múltipla & 3 & 3,6 & 13 & 7,4 & 0,57 & $0,20-1,59$ & 0,23 \\
\hline Assistência pré-natal & 49 & 71,0 & 79 & 59,4 & 1,42 & $0,92-2,19$ & 0,10 \\
\hline Via de parto cesariano & 7 & 8,4 & 64 & 36,6 & 0,24 & $0,12-0,50$ & $<0,01$ \\
\hline \multicolumn{8}{|l|}{ Óbito } \\
\hline tardio & 73 & 88,0 & 106 & 60,6 & 3,22 & $1,76-5,90$ & $<0,01$ \\
\hline intraparto & 6 & 7,2 & 24 & 13,7 & 0,59 & $0,28-1,24$ & 0,13 \\
\hline \multicolumn{8}{|l|}{ Idade gestacional (semanas) } \\
\hline$\geq 37$ & 26 & 31,3 & 29 & 16,6 & 1,68 & $1,17-2,40$ & $<0,01$ \\
\hline$>40$ & 9 & 10,8 & 1 & 0,6 & 3,01 & $2,27-3,99$ & $<0,01$ \\
\hline \multicolumn{8}{|l|}{ Peso $(g)$} \\
\hline$<2500$ (baixo peso) & 48 & 57,8 & 143 & 81,7 & 0,48 & $0,34-0,67$ & $<0,01$ \\
\hline$>4000$ (macrossomia) & 6 & 7,1 & 2 & 1,1 & 2,43 & $1,57-3,78$ & 0,01 \\
\hline
\end{tabular}

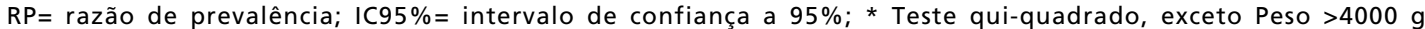
(macrossomia) e Idade gestacional >40 semanas (teste de Fisher); ** Ausente 76 registros. 
adas à indução do parto, as fetais $(p=0,04)$ e anexiais $(p<0,01)$, principalmente o descolamento prematuro da placenta $(p<0,01)$. Enquanto, causas fetais apresentaram maior frequência nas pacientes submetidas à indução do parto, o descolamento prematuro da placenta apresentou baixa frequência (Tabela 3 ).

Após análise multivariada, observou-se que a idade gestacional acima da $40^{\mathrm{a}}$ semana de gravidez $(p<0,01)$ e o óbito fetal tardio $(p<0,01)$ foram os únicos fatores de risco que permaneceram associados à realização da indução do parto. Enquanto, que a via de parto cesariana $(p<0,01)$, funiculopatias $(p=0,02)$, presença de uma ou menos gestações anteriores $(p=0,01)$ e um ou mais partos cesarianos anteriores $(p<0,02)$ foram fatores de proteção, os quais permaneceram associados a não indução do parto (Tabela 4).

Tabela 3

Causas do óbito fetal, segundo a necessidade de indução do parto, em gestantes do Hospital Barão de Lucena, Recife, no período de 2005 a 2008.

\begin{tabular}{|c|c|c|c|c|c|c|c|}
\hline \multirow{3}{*}{ Causas do óbito fetal } & \multicolumn{4}{|c|}{ Indução do parto } & \multirow{3}{*}{$\mathbf{R P}$} & \multirow{3}{*}{ IC95\% } & \multirow{3}{*}{$p^{*}$} \\
\hline & \multicolumn{2}{|c|}{$\operatorname{Sim}(N=83)$} & \multicolumn{2}{|c|}{ Não (N=175) } & & & \\
\hline & $\mathrm{n}$ & $\%$ & $\mathrm{n}$ & $\%$ & & & \\
\hline Indeterminada $(\mathrm{N}=258)$ & 27 & 32,5 & 56 & 32,0 & 1,02 & $0,69-1,48$ & 0,93 \\
\hline Causas maternas & 43 & 75,4 & 86 & 71,7 & 1,14 & $0,69-1,89$ & 0,59 \\
\hline Síndromes hipertensivas & 22 & 38,6 & 55 & 45,8 & 0,82 & $0,52-1,27$ & 0,36 \\
\hline Infecções & 6 & 10,5 & 12 & 10,0 & 1,04 & $0,52-2,07$ & 0,91 \\
\hline Diabetes & 3 & 5,3 & 4 & 3,3 & 1,35 & $0,56-3,26$ & 0,40 \\
\hline Causas fetais & 10 & 17,5 & 9 & 7,5 & 1,77 & $1,08-2,88$ & 0,04 \\
\hline Malformações congênitas & 8 & 14,0 & 8 & 6,7 & 1,64 & $0,95-2,83$ & 0,11 \\
\hline Causas anexiais & 15 & 26,3 & 69 & 57,5 & 0,39 & $0,24-0,66$ & $<0,01$ \\
\hline Descolamento prematuro da placenta & 5 & 8,8 & 39 & 32,5 & 0,29 & $0,12-0,68$ & $<0,01$ \\
\hline Funiculopatias & 2 & 3,5 & 11 & 9,2 & 0,46 & $0,13-1,67$ & 0,15 \\
\hline Síndrome transfusor-transfudido & 1 & 1,8 & 4 & 3,4 & 0,61 & $0,10-3,57$ & 0,48 \\
\hline
\end{tabular}

RP= razão de prevalência; IC95\%= intervalo de confiança a 95\%; * Teste qui-quadrado, exceto Diabetes, Funiculopatias e Síndrome transfusor-transfudido (Teste exato de Fisher).

Tabela 4

Fatores que permaneceram associados à indução do parto, após análise multivariada, em gestantes com óbito fetal do Hospital Barão de Lucena, Recife, no período de 2005 a 2008.

\begin{tabular}{lccc}
\hline Fatores & $\begin{array}{c}\text { Risco ajustado } \\
\text { (Odds Ratio) }\end{array}$ & IC95\% & $p$ \\
\hline Via de parto: cesariana & 0,02 & $0,004-0,09$ & $<0,001$ \\
Causa anexial: funiculopatias & 0,12 & $0,02-0,68$ & 0,02 \\
Gestações anteriores $\leq 1$ & 0,34 & $0,14-0,81$ & 0,01 \\
Idade gestacional > 40 semanas & 126,95 & $3,83-4.201,53$ & 0,007 \\
Cesariana anterior $\geq 1$ & 0,16 & $0,04-0,71$ & 0,02 \\
Óbito fetal tardio & 6,86 & $2,55-18,47$ & $<0,001$ \\
Constante & - & - & 0,16 \\
\hline
\end{tabular}




\section{Discussão}

Atualmente, observa-se crescente utilização do misoprostol para indução do parto, como observado neste estudo. Seu emprego vem sendo consagrado na literatura tanto em gestações com feto vivo quanto em portadoras de óbitos fetais. ${ }^{3-5,7,11-13}$ A dose mais elevada, frequentemente utilizada para indução em fetos mortos, ${ }^{11-13}$ poderia estar relacionado com menor preocupação dos obstetras em deflagrar taquissistolias e/ou sofrimento fetal agudo, causas importantes de óbitos intra-útero quando diagnosticadas tardiamente.

Em nossa amostra, a média da idade materna foi semelhante a outros estudos de base hospitalar.7,15-17 A média da idade gestacional no grupo de gestantes não submetidas aos métodos de indução também foi semelhante a estudo realizado em São Paulo, Brasil, 7 porém maior quando comparado às pacientes que necessitaram do procedimento. É possível que o diagnóstico do óbito intra-útero nas gestações avançadas tenha sido mais precoce devido a melhor percepção da mulher frente a sinais sugestivos e da facilidade de obtenção do diagnóstico, em unidades de alto risco, sob condições de internamento. Ressalta-se ainda que o óbito fetal diagnosticado precocemente necessite mais frequentemente de indução.

Em idades gestacionais mais precoces, o diagnóstico pode ser tardio havendo tempo para que o trabalho de parto se deflagre espontaneamente. Destaca-se frequência elevada de óbitos fetais tardios neste estudo, assim como o peso fetal que foi maior nas pacientes que realizaram indução do parto. Nas pacientes que não foram submetidas ao procedimento, o peso ao nascer foi semelhante a outro estudo.7

Semelhante a outras pesquisas de base hospitalar realizadas no Brasil, 7,15,20,21 o principal método de indução de trabalho de parto empregado foi o uso do misoprostol. Em contrapartida, nenhuma gestante foi submetida ao emprego da sonda de Foley, usualmente indicada em pacientes com história anterior de cesárea. 4

Observa-se que das 258 pacientes incluídas no estudo, $38(14,7 \%)$ relataram uma ou mais cesarianas anteriores e que a maior parte dessas gestantes $(\mathrm{n}=33 ; 86,4 \%)$ não foi submetida à indução do trabalho de parto. A falta de experiência dos médicos assistentes na aplicação de método mecânico para indução nesse grupo de pacientes pode ter influenciado na alta frequência de cesáreas quando comparada a outros estudos. 7 Em São Paulo, Brasil a incidência de partos cirúrgicos foi de apenas $9,1 \%{ }^{7}$
Outro importante fator que pode ter contribuído para o aumento de cesarianas foi a presença de gestantes com descolamento prematuro de placenta (DPP) $(49,3 \%)$, importante urgência obstétrica. 22 Admite-se que a maternidade do HBL, referência em gestações de alto risco no Estado de Pernambuco, receba, por livre demanda ou encaminhamentos de outras unidades de saúde, pacientes mais graves com potencial risco de desenvolverem tal agravo.

As principais causas de óbitos fetais evidenciadas foram as síndromes hipertensivas da gestação, DPP e infecções maternas, semelhante a pesquisas realizadas em São Paulo ${ }^{7}$ e Minas Gerais, Brasil. ${ }^{20}$ Autores ${ }^{20}$ sugerem possível associação entre primiparidade e óbito intra-útero. Há evidência que gestantes primíparas são mais susceptíveis a doenças maternas associadas como pré-eclâmpsia. 23 Autores 20 sugerem associação entre óbito intra-útero e complicações maternas como diabetes, hipertensão e colagenoses, independente da realização da indução do parto. Neste estudo essa associação não foi observada, pois se objetivou determinar fatores associados à indução do parto em fetos mortos. Assim, sugere-se que o procedimento de indução seja ou não indicado independente do diagnóstico materno.

Neste estudo foi observado que o antecedente de uma ou menos gestações anteriores foi fator de proteção para indução do trabalho de parto, ou seja, a indução ocorreu com menor frequência nessas mulheres. Tal resultado está em desacordo com a literatura a qual sugere que o trabalho de parto espontâneo seja desencadeado com maior facilidade em pacientes multíparas. Não se encontrou uma justificativa plausível para essa observação.

Não foram observadas associações entre a indução do trabalho de parto e o momento do óbito fetal, contudo a maior parte das mortes ocorreram no período anteparto - semelhantemente a estudo que encontrou uma frequência de $90 \%$ nesta ocasião, 20 porém, a frequência encontrada foi superior à observada em outro estudo realizado no Brasil.21

As frequências das causas desconhecidas foram semelhantes à literatura disponível. Estima-se que em $12 \%$ a $50 \%$ de perdas fetais a causa da morte não possa ser determinada. A maioria dos autores reconhece a importância da necropsia do feto e estudo anatamopatológico da placenta na elucidação da etiologia do óbito. ${ }^{18}$ Lamentavelmente, em nosso serviço, a necropsia não é realizada de rotina nessas pacientes.

Ressaltam-se ainda outras limitações deste estudo. A coleta retrospectiva de informações por meio de fontes secundárias favorecendo perda de 
respostas para algumas variáveis, caindo sobre um importante viés de informação. Assim, sugere-se a realização de novos estudos prospectivos que possam melhorar a validade interna deste estudo, assim como inferir causalidade já que estudos seccionais servem mais propriamente para gerar hipóteses.

É importante salientar que a análise das carac-

\section{Referências}

1. MacDorman MF, Menacker F, Declercq E. Cesarean birth in the United States: epidemiology, trends, and outcomes. Clin Perinatol. 2008; 35: 293-307.

2. Nicholson JM, Parry S, Caughey AB, Rosen S, Keen A Macones GA. The impact of the active management of risk in pregnancy at term on birth outcomes: a randomized clinical trial. Am J Obstet Gynecol. 2008; 198: 511.e1-15.

3. Souza ASR, Amorim MMR, Costa AAR, Noronha Neto C, Feitosa FEL. O uso do misoprostol para indução do trabalho de parto. Femina. 2010; 38: 127-34

4. Souza ASR, Costa AAR, Coutinho I, Noronha Neto C, Amorim MMR. Análise crítica dos métodos não farmacológicos de indução do trabalho de parto. Femina. 2010; 38: 195-201.

5. Souza ASR, Amorim MMR, Noronha Neto C. Métodos farmacológicos de indução do trabalho de parto: qual o melhor?. Femina. 2010; 38: 277-87.

6. American College of Obstetricians and Gynecologists ACOG Practice Bulletin No 107. Induction of labor. Obstet Gynecol. 2009; 114: 386-97.

7. Aquino MMA, Guedes AC, Mesquita MRS, Hernandez M Cecatti JG. Conduta obstétrica no óbito fetal. Rev Bras Ginecol Obstet. 1998; 20: 145-9.

8. Moraes Filho OB, Cecatti JG, Feitosa FEL. Métodos para indução do parto. Rev Bras Ginecol Obstet. 2005; 27: 493 500 .

9. Kelly AJ, Kavanagh J, Thomas J. Vaginal prostaglandin (PGE2 and PGF2 $\alpha$ ) for induction of labour at term (Cochrane Review). In: The Cochrane Library, Issue 3; 2009. Oxford: Update Software.

10. Souza ASR, Amorim MMR, Feitosa FEL. Comparison of sublingual versus vaginal misoprostol for the induction of labour: a systematic review. BJOG. 2008; 115: 1340-9.

11. Nakintu N A comparative study of vaginal misoprostol and intravenous oxytocin for induction of labour in women with intra uterine fetal death in Mulago Hospital, Uganda. Afr Health Sci. 2001; 1: 55-9.

12. Väyrynen W, Heikinheimo O, Nuutila M. Misoprostol-only versus mifepristone plus misoprostol in induction of labor following intrauterine fetal death. Acta Obstet Gynecol Scand. 2007; 86: 701-5.

Recebido em 11 de março de 2010

Versão final apresentada em 11 de fevereiro de 2011

Aprovado em 1 de abril de 2011 terísticas de gestações com óbito fetal associadas à indução do parto fornece informações valiosas para a formulação de políticas de saúde. O conhecimento desses fatores ajudará na construção de programas para redução de cesariana, particularmente, nas pacientes com óbito fetal e, consequentemente, a sua prevenção e controle.
13. Nyende L, Towobola OA, Mabina MH. Comparison of vaginal and oral misoprostol, for the induction of labour in women with intra-uterine foetal death. East Afr Med J. 2004; 81: 179-82

14. Sampaio ZS, Alencar Júnior CA, Feitosa FEL, Amorim MMR. Fatores associados ao parto vaginal em gestantes de alto risco submetidas à indução do parto com misoprostol. Rev Bras Ginecol Obstet. 2004; 26: 21-9.

15. Guerra GV, Cecatti JG, Souza JP, Faúndes A, Morais SS, Gülmezoglu AM, Parpinelli MA, Passini R, Carroli G, World Health Organization 2005 Global Survey on Maternal and Perinatal Health Research Group. Factors and outcomes associated with the induction of labour in Latin America. BJOG. 2009; 116: 1762-72.

16. WHO (World Health Organization). Recommended definitions, terminology and format for statistical tables related to the perinatal period and use of a new certificate for causa of perinatal deaths. WHO/FIGO. Acta Obstet Gynecol Scand. 1997; 56: 247-53.

17. Petitti DB. The epidemiology of fetal death. Clin Obstet Gynecol. 1987; 30: 253-8.

18. Zlatinik FJ. Management of fetal death. Clin Obstet Gynecol. 1986; 29: 220.

19. OMS (Organização Mundial da Saúde). Classificação Estatística Internacional de Doenças e Problemas Relacionados à Saúde, Décima Revisão (CID 10). 8 ed. São Paulo: Ed. Universidade de São Paulo; 2000.

20. Nurdan N, Mattar R, Camano L. Óbito fetal em microrregião de Minas Gerais: causas e fatores associados. Rev Bras Ginecol Obstet. 2003; 25: 103-7.

21. Almeida MF, Alencar GP, Novaes HMD, França Jr I, Siqueira AAF, Campbell OMR, Schoeps D, Rodrigues LC. Risk-factors for antepartum fetal deaths in the city of São Paulo, Brazil. Rev Saúde Pública. 2007; 41: 35-43.

22. Camano L, Souza E. Assistência ao parto e tocurgia. Manual de orientação da FEBRASGO. São Paulo: Ponto; 2002.

23. Amorim MMR, Souza ASR, Katz L, Coutinho I. Predição da pré-eclâmpsia baseada em evidências. Femina. 2008; 36 : 626-36. 